\title{
Advances in Bispecific Antibodies Engineering: Novel Concepts for Immunotherapies
}

\author{
Ivana Spasevska ${ }^{1,2}$, Minh Ngoc Duong ${ }^{1}$, Christian Klein ${ }^{3}$ and Charles Dumontet ${ }^{1,4^{*}}$ \\ ${ }^{1}$ INSERM 1052/CNRS 5286/UCBL - Cancer Center Research of Lyon - 69008, Lyon, France \\ ${ }^{2}$ Department of Biology, Ecole Normale Supérieure de Lyon - 69342, Lyon, France
}

${ }^{3}$ Roche Pharmaceutical Research and Early Development, Oncology Discovery \& Translational Area, Roche Innovation Center Zurich, Wagistrasse 18, CH-8952 Schlieren, Switzerland

${ }^{4}$ Hospices Civils de Lyon - 69495, Pierre Bénite, France

*Corresponding author: Charles Dumontet, Hospices Civils de Lyon - 69495, Pierre Bénite, France, Tel: + 33 (0) 478777123 ; Fax: + 33 (0) 478777088 ; E-mail: Charles.dumontet@chu-lyon.fr

Received date: Aug 21, 2014, Accepted date: Jan 08, 2015, Publication date: Jan 15, 2015

Copyright: (c) 2015 Spasevska I, et al. This is an open-access article distributed under the terms of the Creative Commons Attribution License, which permits unrestricted use, distribution, and reproduction in any medium, provided the original author and source are credited.

\begin{abstract}
Bispecific antibodies are emerging as novel approach for immunotherapies by combining two antigen-recognizing elements into a single construct that is able to simultaneously bind to two distinct targets. Bispecific antibodies can be applied to recruit immunological effector cells for killing of tumor cells or to simultaneously block two signaling pathways or cytokines. Subsequently, they have prompted significant interest for a number of therapeutic applications, both in cancer and in other indications. The growing interest in therapeutic antibodies along with the rapid progress in antibody engineering have yield a multitude of bispecific antibodies formats and derived molecules that differ in size, shape and function. Presently, two major classes of bispecific antibodies are most widely studied: immunoglobulin-like and small single chain Fv (scFv)-based bispecific antibodies. This review summarizes selected key methods used to generate bispecific antibodies, reports new concepts developed for immunotherapy and discusses their potential development and therapeutic benefits.
\end{abstract}

Keywords: Bispecific antibodies; Immunotherapy; Antibody engineering; IgG-like bispecific antibodies; scFv bispecific antibodies

\section{Introduction}

Monoclonal antibodies (mAbs) have significantly improved the treatment of a variety of human diseases, in particular neoplastic and inflammatory diseases. The best described mechanisms of action of therapeutic monoclonal antibodies include antibody-dependent cellmediated cytotoxicity (ADCC), complement-dependent cytotoxicity (CDC), antibody-dependent cellular phagocytosis (ADCP), (apoptotic) cell death induction, and engagement of cell surface receptors to either activate or inhibit signaling pathways (Figure 1). Currently, the vast majority of antibodies approved for therapy are monospecific, with a defined specificity for a particular molecular part of an antigen, known as an epitope [1]. However, despite being one of the most widely used forms of passive cancer immunotherapy, monoclonal antibodies have not been as successful as expected. Clinical studies have shown that many patients do not adequately respond to monospecific therapy, and resistance to treatment or tumor recurrence is often observed $[2,3]$. This is perhaps not so surprising considering the fact that cancer is often a multifactorial disease, where tumors acquire a series of mutations. Furthermore, many tumors rely on multiple and often redundant pathways of survival and proliferation, as well as cross-talk between signaling cascades. Aiming a single target therefore does not appear to be sufficient to destroy cancer cells.

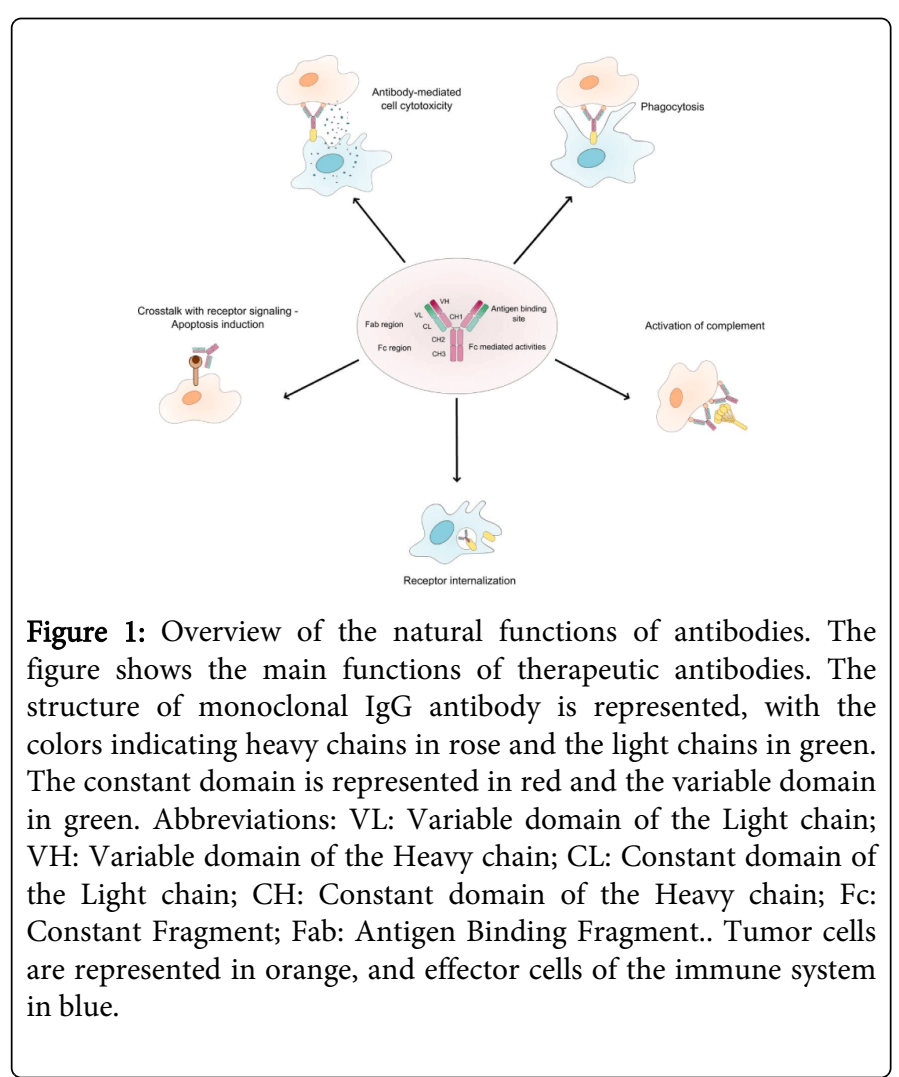


The need to improve the clinical efficacy of antibodies has led to an increased interest in bispecific antibodies (bsAbs). Bispecific antibodies are proteins capable of simultaneously binding two different epitopes, on the same or on different antigens. By simultaneous recognition of two different targets, bsAbs can inhibit two signaling pathways by targeting two different receptors on the same cell or by targeting two different ligands simultaneously; and thus induce modifications of cell signaling, including the inactivation of proliferation or inflammatory pathways. By recognizing two cell surface molecules expressed on different cells bsAbs can also serve as mediators to redirect immune effector cells, such as Natural Killer (NK) cells or T-cells, to tumor cells, in order to enhance the destruction of the latter.

Even though bispecific antibodies have properties similar to those of monoclonal antibodies, they cannot be produced by normal B-cells. Bispecific antibodies can be obtained by different biochemical methods such as chemical conjugation of two antibodies, fusion of two antibody-producing cell lines, or genetic engineering approaches resulting in recombinant bispecific antibody molecules. Currently, two major classes of bispecific antibodies are widely studied: immunoglobulin-G (IgG)-like bsAbs and small single chain Fv (scFv)based bsAbs.

The IgG-like bispecific antibodies have a conserved immunoglobulin constant domain, thus they can exhibit Fc-mediated effector activities and IgG-like pharmacokinetic properties through binding to the neonatal FcRn receptor. Although these antibodies share the same properties as conventional IgG antibodies, a plethora of formats differing in size and geometry have recently been described [4]. Notably, a significant effort has been made to produce bsAbs with an IgG design that is as close to the natural immunoglobulin equivalent as possible. On the other hand, small scFv-based or diabody based bsAbs are genetically engineered recombinant antibodies lacking a constant domain that can be easily engineered and produced, but exhibit short half-life. They have been primarily designed for use as effector cell recruiters, in particular as T-cell engagers. Indeed, scFvbased have been demonstrated to induce lytic immunological synapses identical to those induced by regular T-cell stimuli, and thus provide promising results for immunotherapeutic applications. Currently, over twenty bispecific antibodies have entered clinical trials, and have opened the path to new and improved applications.

This review summarizes a selected number of key techniques and methods to generate bispecific antibodies, reports new concepts developed for cancer immunotherapy, and discusses the perspectives for bispecific antibodies.

\section{The Design and Engineering of IgG-like Heterodimeric Bispecific Antibodies}

Several approaches to generate IgG-like heterodimeric bispecific antibodies have been developed [5].

\section{Quadroma (Hybrid Hybridoma) approach}

The quadroma or hybrid hybridomas was the first approach used to create bispecific antibodies, when the idea for bispecific antibodies arose in the 1980s. The quadroma technology is based on the production of bispecific antibodies by somatic fusion of two hybridoma cells (each being obtained by the somatic fusion of an antibody-producing lymphocyte and a myeloma cell). Each type of hybridoma cells expresses monoclonal antibodies with different specificities. The quadroma cell line from the two fused hybridomas secretes the two antibodies including the bispecific antibody with two distinct arms [6,7] (Figure 2A). However, this method has not been adequate to produce bispecific antibodies in amounts required for therapeutic use, nor in terms of purity because of random chain association.

The random pairing of the light and heavy Ig chains resulted in less than $1 / 10$ of functional bsAbs, with a cumbersome bsAbs purification step. To overcome this chain association issue a chimeric quadroma has been created from a murine and a rat hybridoma cell lines [8]. This chimeric quadroma expressing mouse IgG2a and rat IgG2b chimeric antibodies brought three major technical improvements: (I) enrichment of functional bsAbs because of preferential intra-species heavy/light chain association; (II) efficient heterologous inter-species heavy chain association; (III) simple purification of the desired bsAbs with a specific affinity for a protein $A$ and ion exchange chromatography.

The bispecific antibodies obtained by this method conserve the structure of conventional IgG antibodies, but have a mouse/rat chimeric Fc part with retained effector function. Therefore these antibodies have also been described as trifunctional or Triomabs ${ }^{\oplus}$. In 2009, catumaxomab (Removab ${ }^{\circledR}$ ) became the first bispecific antibody approved for therapeutic usage in Europe. Catumaxomab is a hybrid murine/rat trifunctional bispecific antibody recognizing EpCAM (epithelial cell adhesion molecule) and CD3 (T-cell cluster of differentiation). It is currently used for intra-peritoneal treatment of malignant ascites in patients with EpCAM-positive carcinomas $[9,10]$.

Treatment with catumaxomab resulted in a significantly prolonged puncture-free and overall survival in comparison to the control group or to paracentesis. Moreover, catumaxomab patients had reduced signs and symptoms of ascites than control patients undergoing paracentesis [11]. Nonetheless, adverse events associated with catumaxomab treatment were frequent, and involved both a cytokine release syndrome and potent immunogenicity since catumaxomab is a murine/rat construct.

\section{"Knobs into Holes" and common light chain approach}

The progress in genetic engineering and in DNA recombinant technology has led to a new strategy to facilitate the heavy chain association in a human antibody backbone - the "knobs-into-holes" $(\mathrm{KiH})$ approach. The "knobs-into-holes" approach is based on the introduction of specific mutations in the Fc part of the two antibody heavy chains enforcing heterodimerization of the two different heavy chains. In one of the heavy chain $\mathrm{CH} 3$ domains referred as the "knob" variant a small amino acid has been replaced with a bulky tryptophan residue whereas in the other heavy chain a hole is formed by mutating three residues permitting the interaction with the "knobs" variant miming a key-lock concept [12,13] (Figure 2B).

Moreover, the heterodimeric Fc part can be further stabilized by introduction of a disulfide bridge. The co-expression of the two variants of the heavy chains is sufficient to allow efficient production of defined bsAbs, with $>90-95 \%$ correct chain association. While homodimerization between the "hole" variants can still occur, homodimerization between the "knobs" variants is typically not observed. Recently, alternative technologies to enforce correct heavy chain heterodimerization by electrostatic steering have been described [14-16]. 


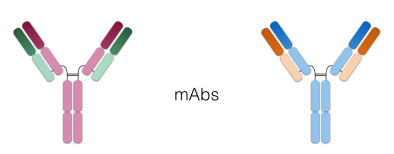

A.

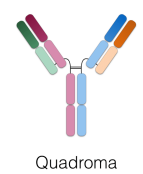

B.
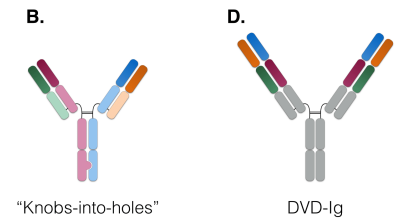

c.

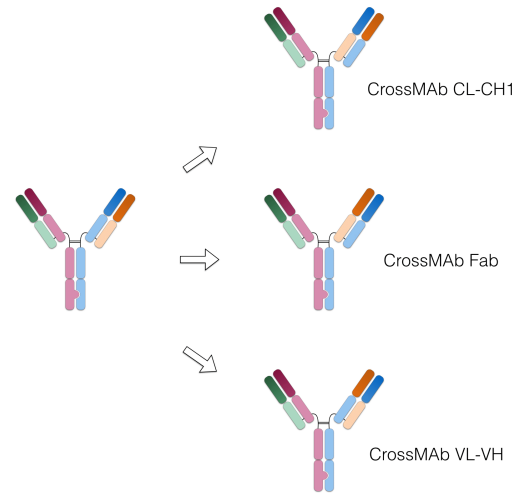

Figure 2: Schematic illustration of different formats of IgG-like bispecific antibodies.The parental monoclonal antibodies are represented above. (A) Heterodimeric functional bispecific antibody obtained with the Quadroma approach. (B) Bispecific antibody designed with the "Knobs-into-holes approach. (C) Bispecific antibody obtained with the CrossMAb approach. The three possible formats are represented with different light chain domains crossover. (D) Dual-Variable-Domain immunoglobulin (DVD-Ig) bispecific antibody with two variable domains.

While the heavy chain association is addressed by the methods discussed previously, it is also essential to impose correct light chain association due to the fact that the light chains have no preference for one or the other of the two heavy chains. One way to solve this problem is to select for antibodies that use a common light chain sequence for antigen recognition [17].

Recently, a bispecific antibody based on the common light chain approach has been described that binds to factors IXa and X and restores factor VIII hemostatic activity in a hemophilia A model [18]. The bispecific antibody is superior to factor VIII in that it does not require thrombin for its activation or von Willebrand factor for stabilization, nor it is affected by factor VIII inhibitors. An optimized version of this antibody is currently in clinical trials for the treatment of hemophilia [15].

Similar to the $\mathrm{KiH}$ approach, an approach based on a set of orthogonal mutations in the Fab domain has recently been described fostering correct light chain assembly [19].

An alternative and elegant strategy for the production of bispecific antibodies without the use of a common light chain is the assembly of two separate antibodies through in vitro processes. This process can be based on two separate antibodies based on the $\mathrm{KiH}$ approach [20] as well as on mutations derived from IgG4 antibodies that foster the annealing of IgG bispecific antibodies [21,22]. Alternatively, a single chain Fab can be used on one arm of the antibody avoiding inappropriate light chain assembly [23].

\section{CrossMAb approach}

The common light chain approach cannot be applied to preexisting antibodies and in many cases no common light chain fulfilling the required properties can be identified at all. Recently, the CrossMAb approach has been described as an option to ensure correct light chain association in bispecific IgG-like antibodies when combined with the "knobs-into-holes" approach. In this structure, one of the antibody's arms is untouched, whereas in the opposite arm both the heavy and the light chain are modified. Three different modifications are possible involving: (I) the entire Fab region; (II) the VL-VH region; (III) the CL-CH1 region [24] (Figure 2C). Consequently, the modified light chain can no longer associate with the unmodified heavy chain; hence, the correct chain association is enforced. Among the three possible models, the CL-CH1 CrossMAb showed the best profile and purity. Importantly, with the CrossMAb method, there is no modification of the antigen-binding specificity, since the only difference with a conventional antibody is the connection to the Fc portion or within the Fab portion [25].

Using this approach, a bispecific antibody CrossMAb CL-CH1 targeting VEGF-A and Ang-2, A2V, was designed [26]. This bsAb is intended to block the ligand-receptor interactions VEGF-A/VEGF receptor 2 and Ang-2/Tunica internal endothelial cell kinase 2 (TIE-2) respectively, and exhibits potent antitumor, antiangiogenic and antimetastatic effects, making it a promising bsAbs for therapeutic applications in cancer therapy and anti-angiogenesis. In preclinical studies, the dual blockade of Ang-2 and VEGF-A by A2V CrossMAb showed anti-tumor effects superior to those observed with either of these factors alone. A2V CrossMAb treatment reduced tumor vessel density, stabilized vessel architecture, and abrogated hypoxia. Consequently, the combination of A2V CrossMAb with chemotherapy led to an improved antitumor efficacy compared to single agent therapies with complete tumor regression in mice. Following these encouraging preclinical data, Ang-2 -VEGF-A CrossMAb is currently in phase 1 clinical trial in patients with solid tumors [27].

\section{Dual-Variable-Domain Immunoglobulin Approach}

The dual-variable-domain Immunoglobulin (DVD-Ig) approach has recently been developed to produce bispecific antibodies. The DVD-Ig bispecific antibodies are generated by combining the variable domains of two pre-existing monoclonal antibodies with different specificities. The variable domains of the two mAbs are fused in tandem via naturally occurring linkers, allowing to create a dual specific IgG-like molecule [28,29] (Figure 2D). The DVD-Ig bsAbs preserve the affinities of both monoclonal antibodies, indicating that each antigen-binding site can function independently without significant steric hindrance. In addition, besides cell surface molecules, the DVD-Ig bsAbs can target soluble molecules, such as interferons, interleukins and chemokines. An optimized DVD-Ig bispecific antibody has many desirable properties such as easy purification to homogeneity using standard approaches, good pharmacokinetic properties, and amenability to large-scale manufacturing [28]. 
Using this approach, the bispecific antibody SAR156597 was generated to target specifically IL-4 and IL-13 in patients with idiopathic pulmonary fibrosis [30]. With a phase 1 of clinical trial recently completed, SAR156597 has recently entered phase 2 trials.

Two other DVD-IgG have also entered clinical trials: ABT-981 simultaneously blocking both IL-1aand IL- $1 \beta$ functions is being studied in phase 1 for osteoarthritis treatment and ABT-122 simultaneously blocking TNFa and IL-17 is being studied in phase 2 for rheumatoid arthritis treatment [31].

\section{The design and Engineering of Small Single Chain Fv - based Bispecific Antibodies}

\section{Bispecific diabodies}

Antibody genetic engineering has led to a production of various antibody fragments differing in size, design and pharmacokinetics, and yet retaining the antigen-binding properties of full-length molecules [32]. Diabodies are a group of small bispecific antibodies generated with DNA recombinant technology, which consist of two $\mathrm{VH}$ and VL domains of two different antibodies. In this design, each VL domain is cross-linked via short peptide linkers with the VH domain of the other antibody (Figure 3A). Since chains from the same antibody tend to dimerize when co-expressed in the same cell, the production of bispecific diabodies requires the following arrangements: VHA-VLB and VHB-VLA (VH-VL configuration) or VLA-VHB and VLB-VHA (VL-VH configuration). Moreover, the linker should be of small size (five amino acids), positioned between the $\mathrm{VH}$ and VL domains in a way that these domains will be forced to associate with the complementary domains of the second antibody. Thus, a diabody is created with two antigen-binding sites [33]. By overexpression of VH-VL fused domains in bacteria (E.coli), soluble diabodies can be produced [34].

Bispecific CD19 x CD16 diabodies were designed to redirect NK cells (CD16+) towards malignant B-cells (CD19+), and thus inducing a specific lysis of tumor cells [35]. Besides NK cells, CD19 $\times$ CD16 bispecific diabodies can recruit other subpopulations of $\mathrm{CD} 16+$ effector cells, such as monocytes and macrophages. The CD19 x CD16 diabodies in combination with CD19 x CD3 bispecific diabodies have demonstrated a synergistic antitumor effect in a preclinical model of non-Hodgkin's lymphoma [36]. Furthermore, CD19 x CD16 diabodies were tested in combination with the angiogenesis inhibitor thalidomide, resulting in significant reduction of tumor size in established human B non-Hodgkin's lymphoma in severe immune deficient mice [37]. Unfortunately, so far no bispecific diabodies have been tested in clinical trials.

Additionally, a new and improved format of diabody was developed, a tetravalent tandem diabody (TandAb) with two binding domains for each target molecule. TandAbs exhibit a superior cytotoxicity and potency relative to diabodies. Two classes of TandAbs have been developed for the recruitment of either T-cells or NK cells, with one antibody of each class in clinical trials: AMF13, a tetravalent CD30 x CD16A NK cell TandAbs currently being tested in phase 2 study for the treatment of Hodgkin's lymphoma [38] and AMF11, a CD19xCD3 T-cell TandAbs in phase 1 clinical trials for nonHodgkin's lymphoma [39].

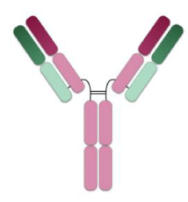

A.

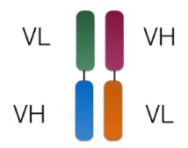

Bispecific diabodies
mAbs

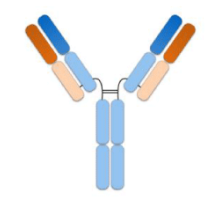

B.

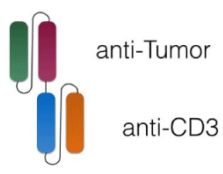

BiTEs
Figure 3: Schematic illustration of different formats of small bispecific antibodies, containing only the variable domains. The parental monoclonal antibodies are represented above. (A) Bispecific diabody with the VH-VL configuration and illustration of the short linker. (B) Bispecific T-cell engager antibodies (BiTEs), with the two different antigen-binging sites binding to $\mathrm{CD} 3$ and to a tumor-related antigen.

\section{Bispecific scFv-based T-cell Engager Antibodies}

An important advantage of bsAbs is the ability of engaging T-cells for tumor cell elimination. Bispecific T-cell engager antibodies (BiTEs) are single chain antibodies designed for polyclonal activation and redirection of cytotoxic T-cells to tumor cells. BiTEs combine the minimal antigen-binding domains of two monoclonal antibodies, fused with a short flexible linker. One of the antibody's arms recognizes $\mathrm{CD} 3$, a cluster of differentiation for T-cells, and the other one detects tumor cells [40] (Figure 3B). The BiTEs antibodies have a high potential to activate T-cells. However, in order to fully activate the T-cell, an interaction between the T-cell and a cluster of BiTEs on the surface of the target cell is necessary. The small design of BiTE antibodies is optimal to enable an interaction between both cells, ensuring the formation of a lytic immunological synapse [41].

Using this approach, blinatumomab (MT103), CD19-specific BiTE antibody has been developed for the treatment of $\mathrm{B}$ cell malignancies [42]. Blinatumomab simultaneously binds CD19-positive malignant B cells and cytotoxic T cells expressing CD3, resulting in efficient T-cellmediated lysis of neoplastic cells. Blinatumomab has shown outstanding results for the treatment of patients with non-Hodgkin's lymphoma and in patients with relapsed or refractory B-precursor acute lymphoblastic leukemia (ALL) [43,44]. Active at very low concentrations, which are easily achievable in the bone marrow microenvironment, blinatumomab treatment demonstrates antileukemic activity in $\mathrm{B}$ cell malignancies. In addition, the elimination of the normal CD19 positive B cells is tolerable. The current standard of care in relapsed or refractory ALL is combination chemotherapy which yields complete remission (CR) in $30-45 \%$ of patients [45]. In a phase 2 study, blinatumomab as a single agent in patients with very advanced disease resulted in more than $70 \%$ CR in the evaluable patients, within two cycles of treatment [46]. Furthermore, with a median follow-up of 8.9 months, $43 \%$ of CR patients remained in remission. The safety profile from the phase 2 trial has shown fever, headache and tremor as the most common 
Page 5 of 8

adverse effects, and a cytokine release syndrome was observed in patients with high tumor burden [46]. Occasional progression of ALL at extramedullary sites (meninges, testes) was also observed, mainly due to insufficient drug penetration. However, with less than $50 \%$ adult ALL patients cured under the best of circumstances and with a high burden of toxicity may contribute to significantly improvement over current therapies. While a phase 3 randomized trial is planned to evaluate chemotherapy with or without blinatumomab in adults with newly diagnosed ALL. (NCT02143414), the US Food and Drug Administration has approved blinatumomab for acute B-cell lymphoblastic leukemia treatment, making it the first bispecific antibody approved in the US [47].

AMG 330, a novel BiTE antibody with characteristics very similar to those previously described for blinatumomab, was developed for the treatment of acute myelogenous leukemia (AML) [48]. AMG 330 has a dual specificity for CD3 and the sialic acid-binding lectin CD33 which is frequently expressed on the surface of AML blasts and leukemic stem cells. Currently in preclinical studies, AMG 330 demonstrated an efficient activation of T-cells along with a potent CD33-dependent cytolytic activity [49,50]. AMG 330 is highly promising for clinical exploration as it may overcome some limitations of previous CD33targeted therapeutics.

MEDI-565 (MT111) is another promising bispecific T-cell engager antibody in development for the treatment of patients with cancers expressing carcino-embryonic antigen (CEA) [51]. A recent study has shown that MEDI-565 may broadly target CEA-positive tumors regardless of the expression of the short splice variant of CEA or SNPs [52]. MEDI-565 has entered phase 1 clinical trials for the treatment of gastrointestinal adenocarcinomas.

The MT110 BiTE antibody recognizes EpCAM, a cell adhesion molecule often expressed in diverse human carcinomas, which was recently shown to promote tumor growth through engagement of elements of the Wnt pathway [53]. In preclinical studies MT110 has demonstrated a highly efficient elimination of colorectal and pancreatic tumor-initiating cells [54,55]. It is currently in phase 1 for diverse human carcinomas including lung cancer, gastric cancer, colorectal cancer, breast cancer, prostate and ovarian cancers.

Immunocore has developed a similar approach comprising an affinity-enhanced soluble T cell receptor (TCR) specific for the HLAA2 restricted melanoma-associated antigen gp100 fused to a CD3 scFv moiety (IMCgp100). Targeting MHC-peptide complexes offers the unique possibility to target not only membrane proteins, but also the intracellular proteome of cancer cells. IMCgp100 is currently being tested in phase 1 clinical trials in melanoma patients $[56,57]$.

\section{Future Perspectives, Limitations and Therapeutic Benefits}

The concept of using bispecific antibodies for cancer immunotherapy was conceived more than 20 years ago, when it became apparent that single targeting is often insufficient to enhance tumor cell destruction. However, the initial clinical studies with bispecific antibodies were rather disappointing due to the low efficacy, stability and immunogenicity. A better understanding of cell biology and immunology and the concomitant development of antibody engineering have led to the production of new classes of bispecific antibodies with better pharmacokinetic properties. The major issue that has been overcome in these past few years has been the ability to produce a pure product on a large scale sufficient for clinical testing, and with satisfactory drug-like properties and stability. Immunogenicity remains a potential issue to be resolved. As is the case for monoclonal antibodies, the immunogenicity of bispecific antibodies depends on the species of origin of antibodies, route of administration, nature of impurities and dosing.

In the case of IgG-like bispecific antibodies, it is important to have an arrangement that is as close as possible to conventional IgG antibodies with fewer linkers or additional domains. Obtaining a closer traditional IgG format should also decrease any possible secondary adverse effects. Although IgG-like bsAbs exhibit appropriate stability, pharmacokinetics and effector functions, their large size may affect tissue penetration. Conversely, small bispecific antibodies may display more efficient penetration, due to their small size. This advantage can be applied in the treatment of solid tumors. However, the small specific antibodies exhibit short half-life and require continuous infusion, and therefore further work needs to be done to increase their serum half-life [58].

With the advances in genetic engineering, bispecific antibodies have experienced a revival and regained the attention of the biopharmaceutical industry. The current engineering methods have yielded over twenty bispecific antibodies currently in clinical trials (Table 1) [59].

\begin{tabular}{|c|c|c|c|c|c|}
\hline INN or code Name & Company & Targets & Type & Conditions & $\begin{array}{l}\text { Clinical } \\
\text { status }\end{array}$ \\
\hline RO5520985 & Roche & Ang2 x VEGF & CrossMAb & Solid tumors & phase 1 \\
\hline AMG212 & Bayer & PSMA $\times$ CD3 & BiTE & ProstaticNeoplasms & phase 1 \\
\hline MT110 & Amgen & EpCAM x CD3 & BiTE & Solid Tumors & phase 1 \\
\hline MT111 & Amgen & CEA $\times$ CD3 & BiTE & MetastaticBreast Cancer & phase 1 \\
\hline IMCgp100 & Immunocore & gp100 x CD3 & ImmTAC & MalignantMelanoma & phase 1 \\
\hline IMP-288 & Immunomedics & CEA $x$ hapten & $\lg \mathrm{g} 1$ & HER2 NegativeBreastCarcinoma & phase 1 \\
\hline ABT-165 & AbbVie & Undisclosed & DVD-Ig & Solid Tumors & phase 1 \\
\hline ABT-981 & AbbVie & & DVD-Ig & Osteoarthritis & phase 1 \\
\hline
\end{tabular}


Page 6 of 8

\begin{tabular}{|l|l|l|l|l|l|}
\hline ACE910 & Chugai & & IgG & Hemophilia & phase 1 \\
\hline AFM11 & Affimed & CD19 $\times$ CD3 & TandAb & NonHodgkin'slymphoma & phase 1 \\
\hline MGD006 & Macrogenics & CD123 $\times$ CD3 & DART & Acute myelogenousleukemia & phase 1 \\
\hline COVA322 & Covagen & TNFa IL17 & FynomAb & RheumatoidArthritis/PsoriaticArthritis & phase 1 \\
\hline AFM13 & Affimed & CD30 $\times$ CD16 & TandAb & Hodgkin'slymphoma & phase 2 \\
\hline FBTA05 & Fresenius, Trion & CD20 $\times$ CD3 & Triomab & B celllymphoma & phase 2 \\
\hline MM-111 & Merrimack & HER2 x HER3 & scFv & HER2 Amplified Breast Cancer & phase 2 \\
\hline & Sanofi & II-4 $\times$ IL-13 & DVD-lg & Pulmonaryfibrosis. & phase 2 \\
\hline ABT-122 & AbbVie & TNF $\times$ IL-12 & DVD-lg & RheumatoidArthritis & phase 2 \\
\hline Blinatumomab & Micromet & C19 $\times$ CD3 & BiTE & Acute lymphoblastic leukemia & Phase 3 \\
\hline
\end{tabular}

Table 1: Selected bispecific antibodies in most advanced phases of clinical studies.

A major benefit from immunotherapies with bispecific antibodies in comparison to conventional therapies is the ability to engage a selective response to the tumor with potent $\mathrm{T}$ cytotoxic cells while limiting off-target toxicity. Targeting T-cells is attractive as they play a key role in immunosurveillance and antitumor immunity, with a remarkable destructive potential. Their engagement is thus essential in cancer immunotherapies. This is an important advantage of bispecific antibodies over classical IgG antibodies, since T-cells do not possess Fc receptors and they cannot be recruited by conventional mAbs [60,61]. Indeed, bispecific antibodies engaging $\mathrm{T}$-cell are the most promising candidates, currently representing the majority of bispecific antibodies in clinical trials. The confirmed efficacy of bispecific antibodies engaging effector T-cells has enhanced the development of new constructs for bsAbs redirecting immune cells to tumor microenvironment. For instance, bsAbs engaging NK cells by targeting CD16 have demonstrated potent tumor cell destruction. The important role of NK cells in tumor microenvironment has been underscored as they significantly contribute to antibody cancer treatment through the ADCC mechanism. Furthermore a combination of T-cell engaging and NK engaging bispecific antibodies resulted in a synergistic effect and an amplified anti-tumor response [36]. The encouraging results obtained by bsAbs engaging T-cells and NK cells are likely to open the path for new designs targeting other immune cells (for example, eosinophils) or tumor microenvironment cells with intrinsic anti-tumor efficacy.

An additional advantage of bispecific antibodies is their ability to block two pathways simultaneously, thereby reducing the risk of resistance (for example, tyrosine kinase receptors and angiogenic ligands). The acquisition of resistance to single agent therapy against one receptor is often associated with pathway switching between two receptors, with a compensatory upregulation and activation of the reciprocal receptor (for example, EGFR and IGFR). Therefore, dual targeting of two receptors simultaneously leads to an improved therapeutic efficacy over combination of monotherapies with mAbs or other therapeutic compounds. In a similar approach, bsAbs have been developed for targeting and neutralizing two soluble factors such as interleukins, interferons and growth factors.

In addition to the use of bispecific antibodies for cancer immunotherapy, bispecific antibodies can be used for virus neutralization (HIV), and as a treatment for inflammatory diseases
$[62,63]$. Recently, new applications for bispecific antibodies have been demonstrated, such as gene-mediated therapy and immunodiagnostic applications [64-66]. Given the encouraging clinical data, improvements in the design and the efficacy of therapeutic bispecific antibodies are likely to continue rapidly.

\section{Conclusion}

This review shows the significant progress that has been made in bispecific antibody engineering and their therapeutic potential. While many challenges remain, these antibodies have promising clinical applications, especially for cancer immunotherapy. Retargeting immune effector cells to tumor cells is an attractive approach with impressive results. Targeting two antigens simultaneously is a promising method in blocking latent tumor escape pathways. However, to completely seize the therapeutic potential of bispecific antibodies, a considerable amount of work is still required to manage the increased complexity involved in their design.

\section{References}

1. Bhutani D, Vaishampayan UN (2013) Monoclonal antibodies in oncology therapeutics: present and future indications. Expert Opin Biol Ther 13: 269-282.

2. Chen LF, Cohen EE, Grandis JR (2010) New strategies in head and neck cancer: understanding resistance to epidermal growth factor receptor inhibitors. Clin Cancer Res 16: 2489-2495.

3. Pandey JP (2012) Mechanism of resistance to cetuximab therapy in colorectal cancer: Possible role of antibodies to immunoglobulin allotypes. MAbs 4: 553-554.

4. Kontermann RE (2012) Dual targeting strategies with bispecific antibodies. MAbs 4: 182-197.

5. Klein C, Sustmann C, Thomas M, Stubenrauch K, Croasdale R, et al. (2012) Progress in overcoming the chain association issue in bispecific heterodimeric IgG antibodies. MAbs 4: 653-663.

6. Suresh MR, Cuello AC, Milstein C (1986) Bispecific monoclonal antibodies from hybrid hybridomas. Methods Enzymol 121: 210-228.

7. Ménard S, Canevari S, Colnaghi MI (1989) Hybrid antibodies in cancer diagnosis and therapy. Int J Biol Markers 4: 131-134.

8. Lindhofer H, Mocikat R, Steipe B, Thierfelder S (1995) Preferential species-restricted heavy/light chain pairing in rat/mouse quadromas. 
Implications for a single-step purification of bispecific antibodies. J Immunol Baltim Md 155: 219-225.

9. Chelius D, Ruf P, Gruber P, Plöscher M, Liedtke R, et al. (2010) Structural and functional characterization of the trifunctional antibody catumaxomab. MAbs 2: 309-319.

10. Linke R, Klein A, Seimetz D (2010) Catumaxomab: clinical development and future directions. MAbs 2: 129-136.

11. Heiss MM, Murawa P, Koralewski P, Kutarska E, Kolesnik OO, et al. (2010) The trifunctional antibody catumaxomab for the treatment of malignant ascites due to epithelial cancer: Results of a prospective randomized phase II/III trial. Int J Cancer 127: 2209-2221.

12. Ridgway JB, Presta LG, Carter P (1996) 'Knobs-into-holes' engineering of antibody $\mathrm{CH} 3$ domains for heavy chain heterodimerization. Protein Eng 9: 617-621.

13. Carter P (2001) Bispecific human IgG by design. J Immunol Methods 248: 7-15.

14. Gunasekaran K, Pentony M, Shen M, Garrett L, Forte C, et al. (2010) Enhancing antibody Fc heterodimer formation through electrostatic steering effects: applications to bispecific molecules and monovalent IgG. J Biol Chem 285: 19637-19646.

15. Sampei Z, Igawa $T$, Soeda $T$, Okuyama-Nishida $Y$, Moriyama $C$, et al. (2013) Identification and multidimensional optimization of an asymmetric bispecific IgG antibody mimicking the function of factor VIII cofactor activity. PLoS One 8: e57479.

16. Von Kreudenstein TS, Escobar-Carbrera E, Lario PI, D'Angelo I, Brault $\mathrm{K}$, et al. (2013) Improving biophysical properties of a bispecific antibody scaffold to aid developability: quality by molecular design. MAbs 5 : 646-654.

17. Jackman J, Chen Y, Huang A, Moffat B, Scheer JM, et al. (2010) Development of a two-part strategy to identify a therapeutic human bispecific antibody that inhibits IgE receptor signaling. J Biol Chem 285: 20850-20859.

18. Kitazawa T, Igawa T, Sampei Z, Muto A, Kojima T, et al. (2012) A bispecific antibody to factors IXa and X restores factor VIII hemostatic activity in a hemophilia A model. Nat Med 18: 1570-1574.

19. Lewis SM, Wu X, Pustilnik A, Sereno A, Huang F, et al. (2014) Generation of bispecific IgG antibodies by structure-based design of an orthogonal Fab interface. Nat Biotechnol 32: 191-198.

20. Spiess C, Merchant M, Huang A, Zheng Z, Yang NY, et al. (2013) Bispecific antibodies with natural architecture produced by co-culture of bacteria expressing two distinct half-antibodies. Nat Biotechnol 31: 753-758.

21. Strop P, Ho WH, Boustany LM, Abdiche YN, Lindquist KC, et al. (2012) Generating bispecific human IgG1 and IgG2 antibodies from any antibody pair. J Mol Biol 420: 204-219.

22. Labrijn AF, Meesters JI, de Goeij BE, van den Bremer ET, Neijssen J, et al. (2013) Efficient generation of stable bispecific IgG1 by controlled Fabarm exchange. Proc Natl Acad Sci U S A 110: 5145-5150.

23. Schanzer JM, Wartha K, Croasdale R, Moser S, Künkele K-P, et al. (2014) A novel glycoenginereed bispecific antibody format for targeted inhibition of EGFR and IGF-1R demonstrating unique molecular properties. J Biol Chem.

24. Schaefer W, Regula JT, Bähner M, Schanzer J, Croasdale R, et al. (2011) Immunoglobulin domain crossover as a generic approach for the production of bispecific IgG antibodies. Proc Natl Acad Sci U S A 108: $11187-11192$

25. Fenn S, Schiller CB, Griese JJ, Duerr H, Imhof-Jung S, et al. (2013) Crystal structure of an anti-Ang2 CrossFab demonstrates complete structural and functional integrity of the variable domain. PLoS One 8: e61953.

26. Kienast Y, Klein C, Scheuer W, Raemsch R, Lorenzon E, et al. (2013) Ang-2-VEGF-A CrossMab, a novel bispecific human IgG1 antibody blocking VEGF-A and Ang-2 functions simultaneously, mediates potent antitumor, antiangiogenic, and antimetastatic efficacy. Clin Cancer Res Off J Am Assoc Cancer Res 19: 6730-6740.
27. Hidalgo M, Tourneau CL, Massard C, Boni V, Calvo E, et al. (2014) Results from the first-in-human (FIH) phase I study of RO5520985 (RG7221), a novel bispecific human anti-ANG-2/anti-VEGF-A antibody, administered as an intravenous infusion to patients with advanced solid tumors. J Clin Oncol 32: 5s.

28. Wu C, Ying H, Grinnell C, Bryant S, Miller R, et al. (2007) Simultaneous targeting of multiple disease mediators by a dual-variable-domain immunoglobulin. Nat Biotechnol 25: 1290-1297.

29. Gu J, Ghayur T (2012) Generation of dual-variable-domain immunoglobulin molecules for dual-specific targeting. Methods Enzymol 502: 25-41.

30. Dhimolea E, Reichert JM (2012) World Bispecific Antibody Summit, September 27-28, 201, Boston, MA. MAbs 4: 4-13.

31. Wu C, Ying H, Bose S, Miller R, Medina L, et al. (2009) Molecular construction and optimization of anti-human IL-1alpha/beta dual variable domain immunoglobulin (DVD-Ig) molecules. MAbs 1: 339-347.

32. Holliger P, Hudson PJ (2005) Engineered antibody fragments and the rise of single domains. Nat Biotechnol 23: 1126-1136.

33. Holliger P, Prospero T, Winter G (1993) "Diabodies": small bivalent and bispecific antibody fragments. Proc Natl Acad Sci U S A 90: 6444-6448.

34. Hornig N, Färber-Schwarz A (2012) Production of bispecific antibodies: diabodies and tandem scFv. Methods Mol Biol 907: 713-727.

35. Kipriyanov SM, Cochlovius B, Schäfer HJ, Moldenhauer G, Bähre A, et al. (2002) Synergistic antitumor effect of bispecific CD19 x CD3 and CD19 x CD16 diabodies in a preclinical model of non-Hodgkin's lymphoma. J Immunol 169: 137-144.

36. Pörtner LM, Schönberg K, Hejazi M, Brünnert D, Neumann F, et al (2012) T and NK cells of B cell NHL patients exert cytotoxicity against lymphoma cells following binding of bispecific tetravalent antibody CD19 $\times$ CD3 or CD19 $\times$ CD16. Cancer Immunol Immunother 61: 1869-1875.

37. Schlenzka J, Moehler TM, Kipriyanov SM, Kornacker M, Benner A, et al. (2004) Combined effect of recombinant CD19 x CD16 diabody and thalidomide in a preclinical model of human B cell lymphoma. Anticancer Drugs 15: 915-919.

38. Reusch U, Burkhardt C, Fucek I, Le Gall F, Le Gall M, et al. (2014) A novel tetravalent bispecific TandAb (CD30/CD16A) efficiently recruits NK cells for the lysis of CD30+ tumor cells. MAbs 6: 728-739.

39. Zhukovsky E, Reusch U, Burkhardt C, Knackmuss S, Fucek I, et al (2013) AFM1, a CD19/CD3 bispecific tandab, to facilitate T-cellmediated killing of CD19+ cells. J Clin Oncol: 3068.

40. Mack M, Riethmüller G, Kufer P (1995) A small bispecific antibody construct expressed as a functional single-chain molecule with high tumor cell cytotoxicity. Proc Natl Acad Sci U S A 92: 7021-7025.

41. Wolf E, Hofmeister R, Kufer P, Schlereth B, Baeuerle PA (2005) BiTEs: bispecific antibody constructs with unique anti-tumor activity. Drug Discov Today 10: 1237-1244.

42. Bassan R (2012) Toward victory in adult ALL: blinatumomab joins in. Blood 120: 5094-5095.

43. Bargou R, Leo E, Zugmaier G, Klinger M, Goebeler M, et al. (2008) Tumor regression in cancer patients by very low doses of a $\mathrm{T}$ cellengaging antibody. Science 321: 974-977.

44. Nagorsen D, Kufer P, Baeuerle PA, Bargou R (2012) Blinatumomab: a historical perspective. Pharmacol Ther 136: 334-342.

45. Gökbuget N, Stanze D, Beck J, Diedrich H, Horst H-A, et al. (2012) Outcome of relapsed adult lymphoblastic leukemia depends on response to salvage chemotherapy, prognostic factors, and performance of stem cell transplantation. Blood 120: 2032-2041.

46. Topp MS, Gökbuget N, Stein AS, Zugmaier G, O'Brien S, et al. (2014) Safety and activity of blinatumomab for adult patients with relapsed or refractory B-precursor acute lymphoblastic leukaemia: a multicentre, single-arm, phase 2 study. Lancet Oncol .

47. Mullard A (2014) FDA approves first bispecific. Nat Rev Drug Discov 14: 7. 
Citation: Spasevska I, Duong MN, Klein C, Dumontet C (2015) Advances in Bispecific Antibodies Engineering: Novel Concepts for Immunotherapies. J Blood Disorders Transf 6: 243. doi:10.4172/2155-9864.1000243

Page 8 of 8

48. Laszlo GS, Gudgeon CJ, Harrington KH, Dell'Aringa J, Newhall KJ, et al. (2014) Cellular determinants for preclinical activity of a novel CD33/CD3 bispecific T-cell engager (BiTE) antibody, AMG 330, against human AML. Blood 123: 554-561.

49. Krupka C, Kufer P, Kischel R, Zugmaier G, Bögeholz J, et al. (2014) CD33 target validation and sustained depletion of AML blasts in longterm cultures by the bispecific T-cell-engaging antibody AMG 330. Blood 123: 356-365.

50. Friedrich M, Henn A, Raum T, Bajtus M, Matthes K, et al. (2014) Preclinical characterization of AMG 330, a CD3/CD33-bispecific T-cellengaging antibody with potential for treatment of acute myelogenous leukemia. Mol Cancer Ther 13: 1549-1557.

51. Osada T, Hsu D, Hammond S, Hobeika A, Devi G, et al. (2010) Metastatic colorectal cancer cells from patients previously treated with chemotherapy are sensitive to T-cell killing mediated by CEA/CD3bispecific T-cell-engaging BiTE antibody. Br J Cancer 102: 124-133.

52. Peng L, Oberst MD, Huang J, Brohawn P, Morehouse C, et al. (2012) The CEA/CD3-bispecific antibody MEDI-565 (MT111) binds a nonlinear epitope in the full-length but not a short splice variant of CEA. PLoS One 7: e36412.

53. Amann M, D'Argouges S, Lorenczewski G, Brischwein K, Kischel R, et al. (2009) Antitumor activity of an EpCAM/CD3-bispecific BiTE antibody during long-term treatment of mice in the absence of T-cell anergy and sustained cytokine release. J Immunother 32: 452-464.

54. Cioffi M, Dorado J, Baeuerle PA, Heeschen C (2012) EpCAM/CD3Bispecific T-cell engaging antibody MT110 eliminates primary human pancreatic cancer stem cells. Clin Cancer Res 18: 465-474.

55. Herrmann I, Baeuerle PA, Friedrich M, Murr A, Filusch S, et al. (2010) Highly efficient elimination of colorectal tumor-initiating cells by an EpCAM/CD3-bispecific antibody engaging human T cells. PLoS One 5: e13474.

56. Oates J, Jakobsen BK (2013) ImmTACs: Novel bi-specific agents for targeted cancer therapy. Oncoimmunology 2: e22891.
57. Liddy N, Bossi G, Adams KJ, Lissina A, Mahon TM, et al. (2012) Monoclonal TCR-redirected tumor cell killing. Nat Med 18: 980-987.

58. Chames P, Van Regenmortel M, Weiss E, Baty D (2009) Therapeutic antibodies: successes, limitations and hopes for the future. $\mathrm{Br} J$ Pharmacol 157: 220-233.

59. Chames P, Baty D (2009) Bispecific antibodies for cancer therapy: the light at the end of the tunnel? MAbs 1: 539-547.

60. May C, Sapra P, Gerber HP (2012) Advances in bispecific biotherapeutics for the treatment of cancer. Biochem Pharmacol 84: 1105-1112.

61. Li J, Zhu Z (2010) Research and development of next generation of antibody-based therapeutics. Acta Pharmacol Sin 31: 1198-1207.

62. Chen W, Xiao X, Wang Y, Zhu Z, Dimitrov DS (2010) Bifunctional fusion proteins of the human engineered antibody domain m36 with human soluble CD4 are potent inhibitors of diverse HIV-1 isolates. Antiviral Res 88: 107-115.

63. Kanakaraj P, Puffer BA, Yao XT, Kankanala S, Boyd E, et al. (2012) Simultaneous targeting of TNF and Ang2 with a novel bispecific antibody enhances efficacy in an in vivo model of arthritis. MAbs 4 600-613.

64. Parashar A, Sarkar S, Ganguly A, Sharma SK, Suresh MR (2011) Bispecific Antibodies for Diagnostic Applications. In: Kontermann RE, editor. Bispecific Antibodies. Springer Berlin Heidelberg: 349-367.

65. Nettelbeck DM (2011) Bispecific Antibodies and Gene Therapy. In: Kontermann RE, editor. Bispecific Antibodies. Springer Berlin Heidelberg: 327-347.

66. van Dieck J, Schmid V, Heind D, Dziadek S, Schraeml M, et al. (2014) Development of bispecific molecules for the in situ detection of proteinprotein interactions and protein phosphorylation. Chem Biol 21: 357-368. 